\title{
Limits on point-like sources with different spectral indexes around the Galactic Centre using the ANTARES neutrino telescope
}

\author{
J. Barrios-Martí \\ Instituto de Física Corpuscular, IFIC (UV-CSIC), Parque Científico, C/Catedrático José Beltrán \\ 2, E-46980 Paterna, Spain \\ E-mail: javier.barrioseific.uv.es
}

on behalf of the ANTARES collaboration

\begin{abstract}
Motivated by an accumulation of events close to the Galactic Centre in the High Energy Starting Events (HESE) reported by the IceCube Collaboration, a search for point-like sources up to an extension of a few degrees in a wide region around the Galactic center has been performed using the ANTARES neutrino telescope. Different spectral indexes for the energy spectra of the sources, in addition to the default value of $\gamma=2.0$, have been tested. Upper limits on the flux normalization as a function $\gamma$ have been set.
\end{abstract}

The 34th International Cosmic Ray Conference,

30 July- 6 August, 2015

The Hague, The Netherlands

${ }^{*}$ Speaker. 


\section{Introduction}

The IceCube collaboration reported an excess of high energy neutrinos which cannot be explained by the expected contribution of atmospheric muons and neutrinos [1], [2]. An accumulation of events is seen in the surroundings of the Galactic Centre. The point with the lowest p-value was found at equatorial coordinates of $(\alpha, \delta)=\left(-79^{\circ},-23^{\circ}\right)$. Although the significance is not enough to identify a point-source, some authors have considered this accumulation could come from a single point-source [3], with an expected flux normalisation of $\Phi_{0}=6 \times 10^{-8} \mathrm{GeVcm}^{-2} \mathrm{~s}^{-1}$. Triggered by this hypothesis, a search for $E^{-2}$ point-sources around the Galactic Center was performed in the last ANTARES[5] point-source analysis [6], with no significant results.

Although the expected energy spectrum for neutrino sources is not completely unkwnown, there is uncertainty on the spectral index. The last HESE analysis results [4] show an expected index of $\gamma=2.50 \pm 0.09$. Herewith, an update of the results of the previous ANTARES analysis around the Galactic Center for different energy spectra (from $\gamma=2.0$ to 2.5 ) is presented. The data sample for this analysis is described in Section 2. The performance of the ANTARES telescope for different energy spectra is shown in Section 3. The procedure of this analysis is explained in Section 4, with the results on Section 5.

\section{Data sample}

The same data sample as in the last published ANTARES point-source analysis is used. The data was collected between January 29, 2007 until December 31, 2012, with a total livetime of 1338 days.

The events in the data sample consist of muon-neutrino source candidates, which are selected following a blind procedure on pseudo-experiments. The selection of the events was tuned to minimise the neutrino flux required for a $5 \sigma$ discovery in $50 \%$ of the experiments for an $E^{-2}$ spectrum. This minimisation was performed by considering different cuts on three parameters of the events: the quality of the track fit, $\Lambda$; the angular error estimate, $\beta$; and the zenith angle, $\theta$. The values of these parameters are obtained by the track reconstruction of the neutrino events, which uses a maximum likelihood (ML) method [7]. The reconstruction is based on a multi-step algorithm to fit the direction of the reconstructed muon by means of a maximisation in the likelihood of the reconstruction. The angular error estimate is later extracted from the estimated uncertainty on the zenith and azimuth angles obtained from the covariance matrix.

A total number of 5516 events are selected for the whole sky in the final sample, with an estimated contamination of mis-reconstructed atmospheric of $10 \%$.

\section{Expected number of events for different energy spectra}

The number of expected signal events which can be detected varies depending on the considered source spectra. For this analysis, power-law spectra of $d \Phi / d E_{v}=\Phi_{0}\left(E_{V} / G e V\right)^{-\gamma}$ are 
assumed. By considering the effective area of the telescope, $A_{e f f}$, given the neutrino energy, $\mathrm{E}_{v}$, and the declination of the source, $\delta$, it is possible to estimate this number as

$$
N(\boldsymbol{\delta}, \gamma)=\int d t \int d E_{v} A_{e f f}\left(E_{v}, \delta\right) \Phi_{0}\left(\frac{E_{v}}{G e V}\right)^{-\gamma}
$$

where the time integration ranges for the whole lifetime of 1338 days. The expected number of signal events for a normalization flux of $\Phi_{0}=10^{-8} \mathrm{GeV}^{-1} \mathrm{~cm}^{-1} \mathrm{~s}^{-1}$ and spectral indices between 2.0 and 2.5 in steps of 0.1 are shown in Figure 1.

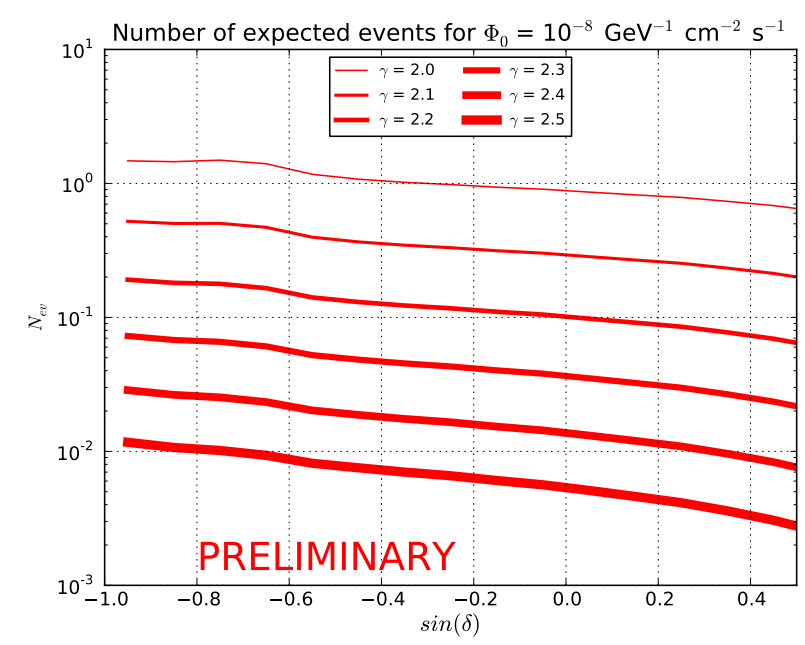

Figure 1: Number of expected signal events as a function of the declination, $\delta$, for energy source spectra between 2.0 and 2.5 in steps of 0.1. A normalization flux of $\Phi_{0}=10^{-8} \mathrm{GeV}^{-1} \mathrm{~cm}^{-1} \mathrm{~s}^{-1}$ has been assumed in all cases.

\section{Search Method}

A search of signal events is performed by means of a maximum-likelihood estimation. This likelihood describes the data in terms of signal and background probability density functions (PDFs). This likelihood is described as,

$$
\log L\left(n_{s}\right)=\sum_{i} \log \left[\frac{n_{s}}{N} S_{i}+\left(1-\frac{n_{s}}{N}\right) B_{i}\right],
$$

where $n_{s}$ indicates the fitted number of signal source events, $N$ is the total number of events in the sample, and $B_{i}$ and $S_{i}$ are the background and signal PDFs for the $i$ th event, respectively. In order to describe the signal and background PDFs, the information of the number of hits, $\mathscr{N}^{\text {hits }}$, angular error estimate, $\beta$, and position in equatorial coordinates, $\vec{x}_{s}=(\alpha, \delta)$, is considered. The signal PDF is described as

$$
S_{i}=\frac{1}{2 \pi \beta_{i}^{2}} \exp \left(-\frac{\psi_{i}\left(\vec{x}_{s}\right)^{2}}{2 \beta_{i}^{2}}\right) P_{s}\left(\mathscr{N}_{i}^{h i t s}, \beta_{i} \mid \gamma\right)
$$


where $P_{S}\left(\mathscr{N}_{i}^{\text {hits }}, \beta_{i} \mid \gamma\right)$ indicates the probability for the $i$ th event to be reconstructed as signal given a number of hits of $\mathscr{N}_{i}^{\text {hits }}$ and an angular error estimate of $\beta_{i}$ for a spectral index of $\gamma$, and $\psi_{i}\left(\vec{x}_{s}\right)^{2}$ represents the angular distance to the assumed source direction, $\vec{x}_{s}$. The distribution of $P_{s}\left(\mathscr{N}^{\text {hits }}, \beta \mid \gamma\right)$ is obtained from simulated events, and it depends on the assumed energy spectra, $\gamma$.

The background PDF is defined as

$$
B_{i}=\frac{B\left(\delta_{i}\right)}{2 \pi} P_{b}\left(\mathscr{N}_{i}^{h i t s}, \beta_{i}\right)
$$

where $B\left(\delta_{i}\right)$ is the probability for an event to be background given its declination, and $P_{b}\left(\mathscr{N}_{i}^{\text {hits }}, \beta_{i}\right)$ is the probability for an to be reconstructed as background with an angular error estimate of $\beta_{i}$ and a number of hits $\mathscr{N}_{i}^{\text {hits }}$. The distribution $B\left(\delta_{i}\right)$ is obtained from the background rate of events from the data sample. The $P_{b}\left(\mathscr{N}_{i}^{\text {hits }}, \beta_{i}\right)$ is obtained also from the information in the data.

In order to determine the significance of any cluster, the test statistic, TS, is defined as TS = $\log L\left(n_{s}\right)-\log L\left(n_{s}=0\right) . L_{b}$ indicates the value of the likelihood where only background events are expected. Larger values of the TS designate a smaller probability of the cluster to be generated from only atmospheric events.

In order to take into account the large uncertainty of the angular error estimates of the IceCube events around the Galactic Center, a search around a region of $20^{\circ}$ around the proposed location $(\alpha, \delta)=\left(-79^{\circ}-23^{\circ}\right)$ is performed. For this purpose, the TS is evaluated in steps of $1^{\circ} \times 1^{\circ}$, while leaving the expected source position, $\vec{x}_{s}$, as a free parameter within these boundaries. In order to estimate the limits, 7 different source declinations were considered in the simulations.

\section{Results}

No significant cluster has been found in the defined area around the Galactic Centre. Figure 2 shows the results presented in the last ANTARES point-source analysis, where different source extensions were considered (point source, $0.5^{\circ}, 1^{\circ}$ and $2^{\circ}$ ). $90 \%$ C.L. upper limits on the flux normalisation, $\Phi_{0}$, for the different assumed source spectra and as function of the declination can be seen in figure 3. Figure 4 shows the limits for a declination of $\delta=-29^{\circ}$. In this figure, the expected flux normalisation from the hypothetical source depending on the number of HESE events which would be originated in this source is also considered. These values have been obtained from [8]. A point-like source with values of the spectral index closer to 2.5 are more disfavoured than for values closer to 2.0 .

\section{Conclusions}

A point-source search around the Galactic Centre for spectral indices between 2.0 and 2.5 with the ANTARES neutrino telescope has been performed. No significant cluster has been found, and $90 \%$ C.L. upper limits have been set. According to these limits, point-like sources with softer spectra are more disfavoured to explain the accumulation of events observed by IceCube. 


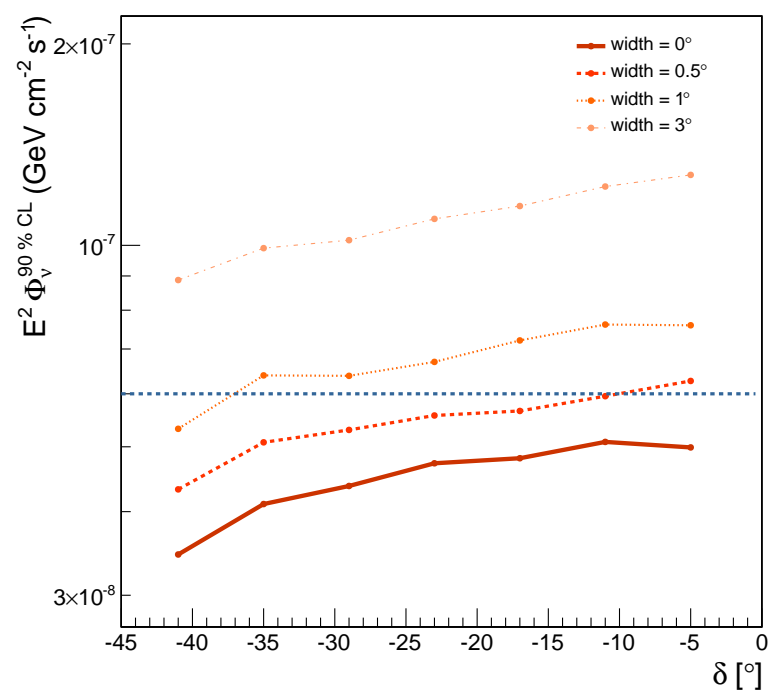

Figure 2: $90 \%$ C.L. upper limits for a point-source and for source extensions of $0.5^{\circ}, 1^{\circ}$ and $2^{\circ}$ as a function of the declination.

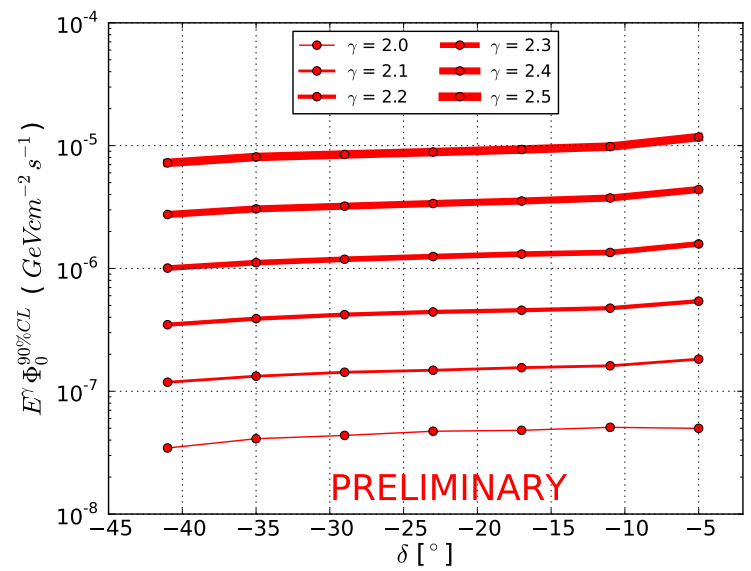

Figure 3: $90 \%$ C.L. upper limits for source spectra between 2.0 and 2.5 as a function of the declination of the source.

\section{Acknowledgments}

The authors acknowledge the support of the Spanish MINECO through project FPA201237528-C02-01, the MultiDark Consolider Project CSD2009-00064, the Generalitat Valenciana via Prometeo-II/2014/079, , and of Universitat de València, Atracció de Talent.

\section{References}

[1] M.G. Aartsen et al, Science 342, 1242856 (2013) 

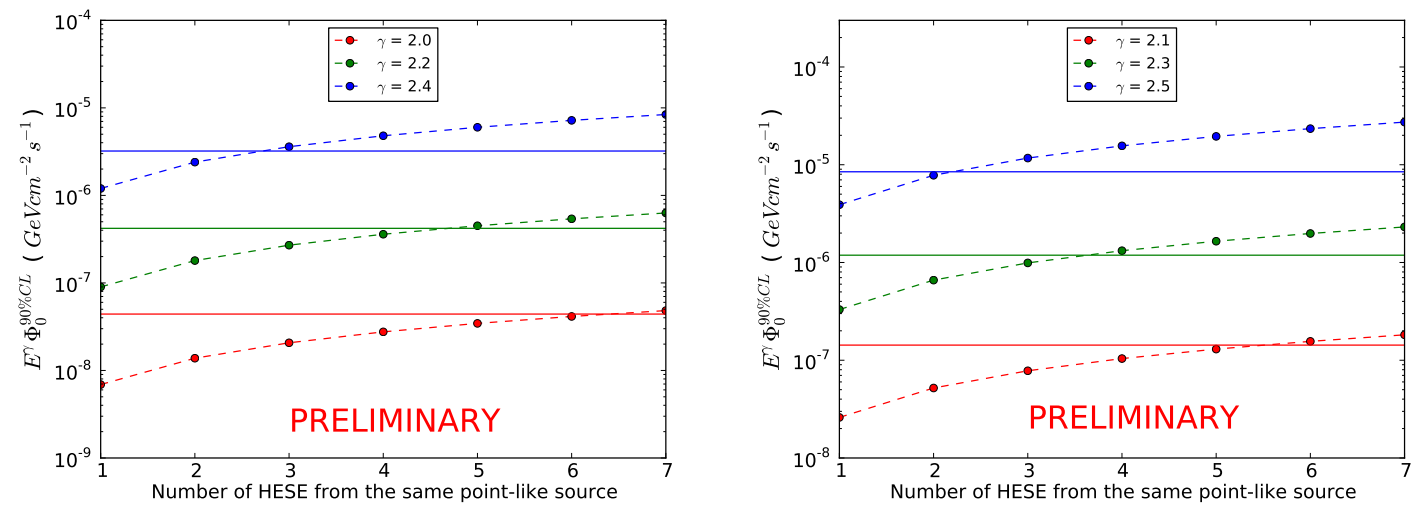

Figure 4: Solid lines: $90 \%$ C.L. upper limits for source spectra between 2.0 and 2.5 and a source declination of $\delta=-29^{\circ}$. The figure on the left contains the values for $\gamma=2.0,2.2$, and 2.4, whereas the figure on the right contains the ones for $\gamma=2.1,2.3$ and 2.5. Dashed lines: expected flux normalisation of the proposed source as a function of the number of HESE events coming from this source. Values above the solid lines are disfavoured with a confidence level larger than $90 \%$.

[2] M.G. Aartsen et al., Phys. Rev. Lett. 113, 101101 (2014)

[3] M.C. González-García, F. Halzen \& V. Niro, Astropart.Phys., 57-58, 39 (2014)

[4] M. G. Aartsen et al, Phys. Rev. D, 91, 022001 (2015)

[5] M. Ageron et al., Nucl. Instrum. Meth. A 656, 11-38 (2011)

[6] S. Adrián-Martínez et al. Astrophysics J., 786, L5 (2014)

[7] S. Adrián-Martínez et al., Astrophysics J., 760, 53 (2012)

[8] M. Spurio, Phys. Rev. D, 90, 103004 (2014) 